\title{
Correlative Imaging and Cryo-FIB Processing for Direct Visualization of HIV-1 Infection
}

\author{
P. Zhang ${ }^{1}$, S. Jun ${ }^{1}$, G. Zhao ${ }^{1}$, Z. Ambrose ${ }^{2}$, and S. C. Watkins ${ }^{3}$
}

1. Department of Structural Biology, School of Medicine, University of Pittsburgh, Pittsburgh,USA

2. Division of Infectious Diseases, Department of Medicine, University of Pittsburgh, Pittsburgh, USA

3. Department of Cell Biology and Physiology, University of Pittsburgh, Pittsburgh, USA

Cryo-electron tomography (cryo-ET) allows 3D visualization of cellular structures at molecular resolution in a close-to-native state, thus has the potential to help elucidate early events of HIV-1 infection in host cells [1]. However, direct observation of structural details of infecting HIV-1 has not been realized due to technological challenges in working with rare and dynamic HIV-1 particles in human cells. Here, we combined two imaging modalities, live-cell fluorescence light microscopy to follow the dynamics of viral particles inside of the cell [2-4] and cryoET for high resolution cellular ultra-structures for HIV-1 and host-cell interactions. Using this methodology, we directly visualized, for the first time under near-native conditions, that intact HIV-1 cores are released into the cytoplasm of host-cells. We further obtained direct evidence to suggest that a hyperstable mutant capsid, E45A, delayed capsid disassembly compared to the wild-type capsid [5].

Another major limitation in cryoET imaging, the accessible specimen thickness, has also hindered direct visualization of HIV particles which have traveled into the deep side of the host cell. Recently several studies have demonstrated the feasibility of cryo-FIB milling to reduce the specimen thickness using small bacterial cells [6-7]. Here we report the technical advances in cryo-FIB processing of large mammalian cells, creating samples suitable for 3D structural analysis. We developed a novel FIB approaches to create thin, freely-suspended, cell lamellas directly from HeLa cells cultured on EM grids, without requiring the technically challenging cryo-FIB "lift-out" procedure to transfer lamellas. We demonstrate our new approaches with high quality 3D cellular structures obtained from native bacterial cells and large mammalian cells [8]. Together, these technological advances will greatly facilitate high resolution imaging of dynamic processes, such as viral infections and cell signaling events [9].

\section{References:}

[1] A. Leis et al., Trends Biochem Sci 34 (2009) 60.

[2] Arhel et al., Nat Methods 3 (2006) 817.

[3] McDonald et al., J Cell Biol 159 (2002) 441.

[4] Koch et al., Retrovirology 6 (2009) 84.

[5] S. Jun et al., Structure 19 (2011) 1573.

[6] M. Marko et al., Nat Methods 4 (2007) 215.

[7] A. Rigort et al., J Struct Biol 172 (2010) 169.

[8] K. Wang et al., J Struct Biol 180(1012) 318.

[9] This research was supported by National Institutes of Health (RR024424 and GM085043). 

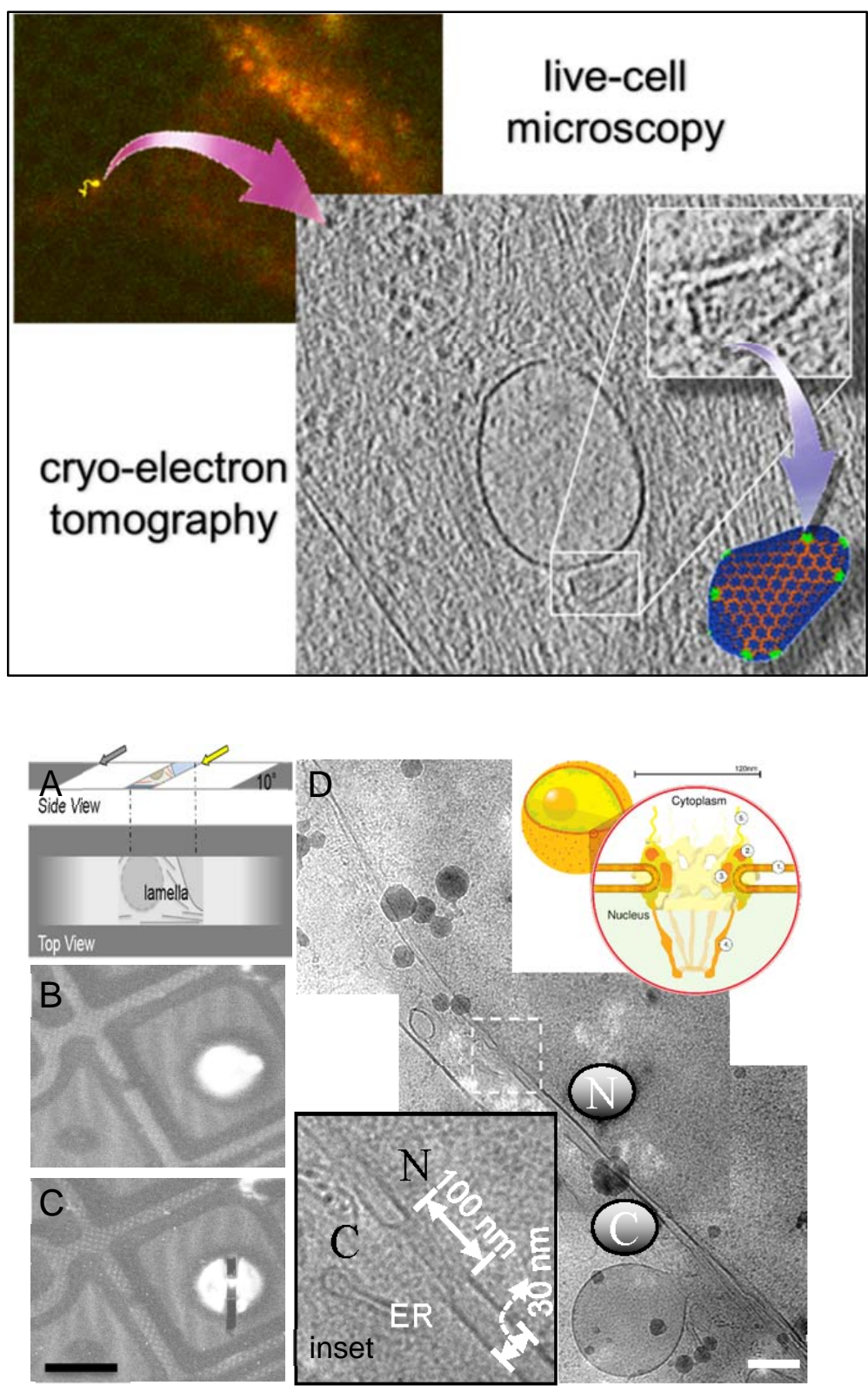

Figure 1. Correlative confocal live-cell and cryoET imaging of a single HIV-1 core released into the $\mathrm{HeLa}$ cell cytoplasm after membrane fusion. The GFP-tagged HIV virion was tracked for 30 minutes for its movement in HeLa cell post-fusion (yellow trace) and then plunge-frozen immediately. The correlative cryo-ET analysis shows the exact same viral particle in the context of surrounding cellular ultrastructure in a 3D cellular tomogram in near native condition. The viral core is clearly visible next to a large vesicle.

Figure 2. Cryo-FIB milling of a HeLa cell lamella. (A) Schematic of cell lamella milling with cryo-FIB. (B\&C) Cryo-SEM images showing a HeLa cell before (B) and after (C) cryo-FIB milling and creation of a cell lamella. (D) Cryo- EM projection images showing the nuclear envelope of the HeLa cell, recorded from the cryo-FIB prepared lamella in Figure 5C. The nucleus is indicated by an $\mathrm{N}$ and the cytoplasm by a C. The inset in Figure $5 \mathrm{D}$ is an enlarged view of the boxed region, revealing a nuclear pore (shown in schematic in upper right). Scale bars: $50 \mu \mathrm{m}(\mathrm{C})$ and $200 \mathrm{~nm}$ (D). 